\title{
Research on the Development of Online Paid Q \& A Platform from the Perspective of Perceived Value
}

\author{
Yihao Zhang*
}

Information Engineering School, Hangzhou Dianzi University, Hangzhou 311300, China. E-mail: 531598369@qq.com

Abstract: After the rise of online paid Q \& A platform in 2016, the development of online paid Q \& A platform has become a bottleneck. In order to promote the development of online paid Q \& A platform, the bottleneck of paid Q $\&$ A platform is analyzed from the perspective of perceived value. The perceived value is enhanced by the technical characteristics of the perceived cost and the usefulness and entertainment of the perceived benefit. Research shows that perceived value can enhance the development of online paid Q \& A platforms. On this basis, this paper puts forward some relevant countermeasures for the development of online payment Q \& A platform.

Keywords: Perceived Value; Knowledge Payment; Online Payment Q \& A Platform

\section{Introduction}

Since the popularization of personal home computer, the concept of free information has also spread, the Internet is full of a large number of disassembled, reorganized fragmented information, disorderly and excessive information can not meet the needs and speed of user knowledge growth. The rise of online paid Q \& A platforms on the Internet in 2016 is helping users get organized and streamlined information by paying for it, as well as generating revenue from their cognitive surplus. But since 2016, the development of online paid Q \& A platforms has fallen into a bottleneck ${ }^{[1]}$. This paper analyzes the development of online paid question answering platform from the perspective of perceived value through a large number of literature research.

\section{The development of online paid question-and-answer platform and the concept of perceived value}

\subsection{The development of online paid Q \& A platform}

The development of online payment Q \& A platform has been divided into several stages as follows: knowledge sharing, knowledge payment germination and knowledge payment development ${ }^{[1]}$.

This phase of knowledge sharing began in 1999 with the launch of the "Look Smart Live" , a web-based interactive question-and-answer platform. From Look Smart in the United States until 2010, a great deal of free information is flooding the Internet, and knowledge sharing is taking the form of open forums and shared platforms.

2011-2015 is regarded as the embryonic stage of knowledge payment. From a handful of forums and sharing platforms, it began to experiment with a paid model -- as a download, a service, a membership, and a paywall. The core difference between knowledge payment and traditional payment is gradually formed, and the characteristic of know-

Copyright (C) 2020 Yihao Zhang

doi: 10.18282/ff.v9i2.877

This is an open-access article distributed under the terms of the Creative Commons Attribution Non-Commercial License

(http://creativecommons.org/licenses/by-nc/4.0/), which permits unrestricted non-commercial use, distribution, and reproduction in any medium, provided the original work is properly cited. 
ledge being valuable is highlighted ${ }^{[2]}$.

In 2016, known as the first year of the development of China's online paid Q \& A platform, the explosive growth of the Himalayan MF, Zhihu Live, Fenda, Weibo Q \& A and so on has made the topic of knowledge payment once again received public attention, thus started the stage of the development of knowledge payment. So far the popularity of online paid Q \& A platforms has passed, but it has become part of our daily lives.

\subsection{Perspective of perceived value}

At present, there are many opinions on the theory of perceived value, and the view of perceived value is based on the theory of user perceived Value Acceptance Model (VAM) proposed by Kim et al. The model of perceived value acceptance is based on the theory of perceived value and the model of technology acceptance. At present, the model of perceived value acceptance is adopted by many scholars.

The perceived value perspective proposed in this paper is based on the perceived benefit and perceived effort of the user perceived value acceptance model (VAM) as the two core perspectives of the perceived value perspective as shown in Figure 1.

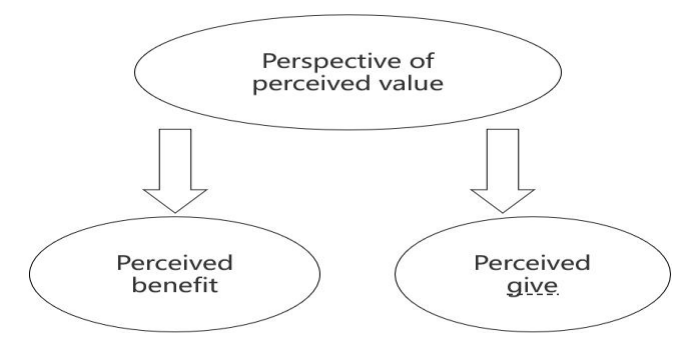

Figure 1. Perspective of perceived value.

The perceived benefit has two factors in the perceived value acceptance model (Vam): Usefulness and entertainment. There are also two perspectives of perceived costs: technical features and perceived $\operatorname{costs}^{[3]}$.

\section{The bottleneck of the development of online paid $Q$ \& A platform from the perspective of perceived valve}

\subsection{The barriers to platform development caused by perceived effort}

The current online paid Q \& A platform does not do enough to reduce the perceived cost to users. Thus, after 2016, the development of online paid Q \& A platform is still restricted by the perceived cost.

In terms of money costs, the current online $\mathrm{Q} \& \mathrm{~A}$ on Weibo, Q \& A, Zhihu Live and other online $\mathrm{Q} \& \mathrm{~A}$ sites are still relatively expensive to ask and watch, although almost all platforms are set by users or respondents, but because the user asks the question and wants to provide the solution the object is the flow big name or the specialized domain big cow, causes the price to remain high. For example, Fan Chengcheng (Fan Bingbing's younger brother) once set the price of a Weibo question and answer session as 50 RMB.

In terms of time cost, the time cost of solving a user's question is much higher than the time cost of getting an answer from a free forum community or search engine (the correctness of the answer is not discussed here). And because respondents can choose whether to answer questions or not, the money they pay to ask questions for more than a day is returned to the questioner, again raising the questioner's cost of time $\mathrm{e}^{[4]}$.

\subsection{The barriers to platform development posed by perceived benefits}

The development of the platform is restricted not only by the perceived effort, but also by the perceived benefit.

The online paid Q \& A platform only provides users with the answers to the traffic and professional responses ${ }^{[5]}$. The platform can not give users a definite correct answer, users can only rely on the respondent's personal social influence to make a subjective judgment of the answer. 
At present, the main force of online Q \& A platform is still entertainment topics, such questions can bring users the perceived benefits of far less than economic, political, military and other topics. Online paid Q \& A platforms can not give users a reason not to pay.

\subsection{The barriers to the development of the platform by the combination of perceived effort and perceived benefit}

There is a negative correlation between perceived cost and perceived benefit, and the greater the perceived cost, the less the perceived benefit. The absolute value of perceived benefit is taken as 100 yuan. When the perceived cost changes from 30 yuan to 50 yuan, the relative value of perceived benefit also decreases. Likewise, when the absolute value of perceived effort is $\$ 100$, the perceived benefit changes from $\$ 50$ to $\$ 30$, and the relative value of perceived effort increases.

For example, from the point of view of the continuous loss of users, the popularity of Weibo Q \& A has grown from its initial popularity to its current calm, which is due to the large amount of attention it received from users when it was launched, no matter what kind of question and answer is provided by Weibo, it is recognized by a large number of users, so the recreational question and answer in people's daily life in the form of after-dinner conversation and other forms of enhancing the users' perception of the benefits ${ }^{[6]}$. With the loss of users and the decline of the popularity of online paid Q \& A platforms, people's perceived benefits declined, while the perceived costs of users remained unchanged and the relative value of perceived costs rose, the loss of users will hinder the development of the platform.

\section{How to enhance the perceived value and better effect on the development of online paid Q \& A platform.}

\subsection{Perceived effort—characteristics of technology}

The difference between the profit and the cost constitutes the perceived value. The cost of the consumer is related to the complexity of the technology and the perceived cost of the product. In general, the more complex the technical characteristics of a product, the more difficult it is for consumers to obtain the information they need and to master the technical aspects of using the product ${ }^{[7]}$.

So far, the online paid Q \& A platform's main target customers are young people, but due to the technical characteristics of the reasons for middle-aged and elderly people with a considerable barrier to entry. The current sub-answer, Zhihu and other online payment Q \& A platforms need to bind payment methods again, if the online paid Q $\&$ A platform can be embedded into the channels of Wechat/Alipay to reduce the re-binding of payment methods and so on can effectively reduce the perception of technical characteristics of middle-aged and elderly people, and increase the use of middle-aged and elderly people, so the platform can be developed through the emerging user ${ }^{[8]}$.

\subsection{Perceived effort-perceived cost}

In this paper, the perceived cost is defined as the sum of the time cost and expense paid by the user for using the online paid Q \& A platform.

When it is in the stage of paying knowledge development, the starting price of 1 yuan has obvious difference compared with the initial stage of paying knowledge. Now four years from the first year of the development of online paid Q \& A platforms, the "1 yuan" price needs to be adjusted again to "0.1 yuan" to stimulate customer perception fees, lower prices from customers in the current situation will help to stimulate user activity.

\subsection{Perceived benefit—usefulness}

Usefulness is the fundamental factor for users to choose to use online paid Q \& A platform. The key difference between online paid Q \& A platform and shared platform is the validity of the information answer ${ }^{[9]}$. The more valid the information answer is, the more valid it is for the user.

A month after the question and answer session, the content of the questions and answers will be released to the rest 
of the audience free of charge. 100,000 questions in 3 days, $75 \%$ likes, bonus from the platform, then buy out in advance and share it for free one week later, it not only ensures the benefit of platform, questioner and answerer, but also increases the user's perceived usefulness.

\subsection{Perceived benefit - entertainment}

Perceived entertainment is the degree to which a user perceives the entertainment value of a product to provide self-satisfaction. The current forms of online paid Q \& A platforms are as follows: micro-blog Q \& A text, Zhihu Live, Foshan voice and so on. Increase the number of respondents, questioners, and onlookers interacting-for example, when a question has a large number of certified domain respondents and a large number of users, instead of a single text-only or voice-only interface, it could be changed through this year's surge in ad-hoc web video conferencing ${ }^{[10]}$.

\section{Conclusion}

Here is ample room for the development of online paid question-and-answer platforms from the perspective of perceived value, and in the future, after the academic community has a unified definition of perceived value theory, online paid Q \& A platforms offer new value for users.

\section{References}

1. Zhang Y. Overview of online platform knowledge payment (in Chinese). Information Research 2018; 8: 129-134.

2. Kang M. Research on the continuous use intention of paid Q \& A platform users (in Chinese). Southwest Jiaotong University; 2019. p. 1-3.

3. Zhang L, Zhou X. Research on trust based on knowledge Payment of Online Question answering platform (in Chinese). Economic Research Guide 2019; 18: 159-161.

4. Li W, Ai P, Bing F. Fan's Power and paid knowledge: Research on the behavior of paying to watch of online question-and-answer platform users (in Chinese). Library Journal 2019; 4.

5. $\mathrm{Li} \mathrm{W}, \mathrm{Xu} \mathrm{Y}$, Cong T. The influence of the perceived value of users on their willingness to pay on online paid Q \& A platform (in Chinese). Press Circles 2018; 10: 94-102.

6. Han F. Factors influencing the willingness to use the paid Q \& A platform (in Chinese). Central China Normal University; 2017. p. 1-3.

7. Yu L. Study on the profit model of Weibo paid Q \& A in the era of cognitive surplus (in Chinese). Central South University for Nationalities; 2018. p. 1-3.

8. Zhao F, Qu Y, Zhou Q. An empirical study on the influencing factors of the willingness to pay for knowledge of community users question and answer community. Information and Documentation Services 2019; 40(1): 89-97.

9. Liu Z, Shi Y. The commercialization of interaction: The influence and significance of the introduction of the payment mechanism into the knowledge Q \& A platform (in Chinese). Modern Communication 2020; 2: 137-141. 